\title{
DO ULTRALUMINOUS X-RAY SOURCES EXIST IN DWARF GALAXIES?
}

\author{
Douglas A. Swartz ${ }^{1}$ Roberto Soria ${ }^{2}$, and Allyn F. Tennant ${ }^{3}$
}

Submitted to Astrophysical Journal

\begin{abstract}
A thorough search for Ultraluminous X-ray source candidates within the Local Volume is made. The search spatially matches potential ULXs detected in X-ray images or cataloged in the literature with galaxies tabulated in the Catalog of Neighboring Galaxies compiled by Karachentsev et al. (2004). The specific ULX frequency (occurrence rate per unit galaxy mass) is found to be a decreasing function of host galaxy mass for host masses above $\sim 10^{8.5} \mathrm{M}_{\odot}$. There is too little mass in galaxies below this point to determine if this trend continues to lower galaxy mass. No ULXs have yet been detected in lowermass galaxies. Systematic differences between dwarf and giant galaxies that may explain an abundance of ULXs in dwarf galaxies and what they may imply about the nature of ULXs are discussed.
\end{abstract}

Subject headings: galaxies: general $-\mathrm{X}$-rays: galaxies $-\mathrm{X}$-rays: general

\section{INTRODUCTION}

Ultraluminous X-ray sources (ULXs) are defined as the most X-ray luminous, typically $L_{\mathrm{X}} \gtrsim 10^{39} \mathrm{ergs} \mathrm{s}^{-1}$, nonnuclear point-like objects in nearby galaxies. What environments and what types of stellar systems give rise to the ULX phenomenon is a subject of considerable topical interest.

The study of ULXs as a class has matured beyond mere number counts. The general approach is to search for statistically-significant correlations between ULXs and properties of their galactic environments. In this way, one hopes to eventually understand what physical processes are responsible for their formation, what influence they have on their environment, and how they have evolved over cosmic time. Studies of ULXs as a class have reached some robust conclusions; ULXs are associated with active star formation, at least on galaxy-wide scales (Swartz et al. 2004; Gilfanov et al. 2004; Liu et al. 2006; Winter et al. 2006) being more common and more luminous in starburst and interacting/merging galaxies (e.g., Zezas et al. 2002, Gao et al. 2003) than in normal galaxies and occurring more frequently in the past (Lehmer et al. 2006) consistent with the observed rise in star formation density with redshift. ULXs are less luminous in early-type galaxies, potentially revealing a secondary population of unusually bright low-mass X-ray binaries or perhaps fortuitous beaming of a fraction of this population towards the observer (Irwin 2004; Swartz et al. 2004; Liu et al. 2006; see also King 2004).

There are circumstantial reasons to suspect that there may be differences between the rate of ULXs in nearby dwarf and giant galaxies. Dwarf galaxies typically lack organized structure like bars and density waves that drive star formation yet their star formation rate (SFR) per unit area can be comparable to those of spiral galaxies (Hunter \& Gallagher 1986). Dwarf galaxies have evolved more slowly, retaining a higher gas fraction than giants in the current epoch (Geha et al. 2006) and resulting in a lower metallicity (Lee et al. 2006 and references therein). Environmental factors, such as ram-pressure stripping, can strongly affect star formation in dwarfs whereas star formation in giant field galaxies is determined more by their merger history (Haines et al. 2007). If ULXs (or the luminous subset of ULXs) are preferentially associated with star formation, then there may be differences in the rates of ULXs in dwarfs compared to giants because of the different internal and environmental influences on star formation activity in galaxies of different mass.

The ULX population in the Local Volume $(D \leq 10 \mathrm{Mpc})$ is investigated in the following. $\S 2$ describes the sample of galaxies included in the study and defines three subsamples with differing selection criteria and hence different sample biases. The results of the search for ULXs in these subsamples are presented in $\S 3$ where it is shown that the specific ULX frequency (number per unit galaxy mass) increases towards lower galaxy mass down to a limit of $\sim 3 \times 10^{8} \mathrm{M}_{\odot}$ below which no ULXs have yet been detected. The significance of this result is discussed in $\S 4$.

\section{THE SAMPLES}

By definition, ULXs are the most luminous non-nuclear sources in galaxies. Nevertheless, their $0.5-8.0 \mathrm{keV}$ flux, $f_{\mathrm{X}}>8.3 \times 10^{-12} / D^{2}$ erg $\mathrm{cm}^{-2} \mathrm{~s}^{-1}$ (for a $L_{\mathrm{X}}=$ $10^{39} \mathrm{ergs} \mathrm{s}^{-1}$ source; the traditional definition of a ULX), quickly falls below detection sensitivities of most X-ray observatories for sources beyond $D \sim 20-30 \mathrm{Mpc}$ when observed for typical exposure times. Similarly, properties of dwarf galaxies are best known for nearby objects. In this case, the galaxies of interest are the least intrinsically luminous and have the smallest physical size. For these reasons, a study of ULXs in dwarf galaxies is currently restricted to nearby objects within roughly the Local Volume. The recent compilation of galaxy properties by Karachentsev et al. (2004, hereafter K04) is purported to be about 70$80 \%$ complete out to a distance $D=8 \mathrm{Mpc}$. The catalog includes galaxies brighter than $B_{t} \sim 17.5 \mathrm{mag}$ and angular sizes larger than $D_{25} \sim 0.4^{\prime}$. This Catalog of Neighboring

\footnotetext{
${ }^{1}$ Universities Space Research Association, NASA Marshall Space Flight Center, VP62, Huntsville, AL, USA

${ }^{3}$ Mullard Space Science Laboratory, University College London, Holmbury St. Mary, Surrey RH5 6NT, UK

${ }^{3}$ Space Science Department, NASA Marshall Space Flight Center, VP62, Huntsville, AL, USA
} 
Galaxies will be referred to as the CNG in this paper and various samples of galaxies and their properties will be taken from the values reported in K04.

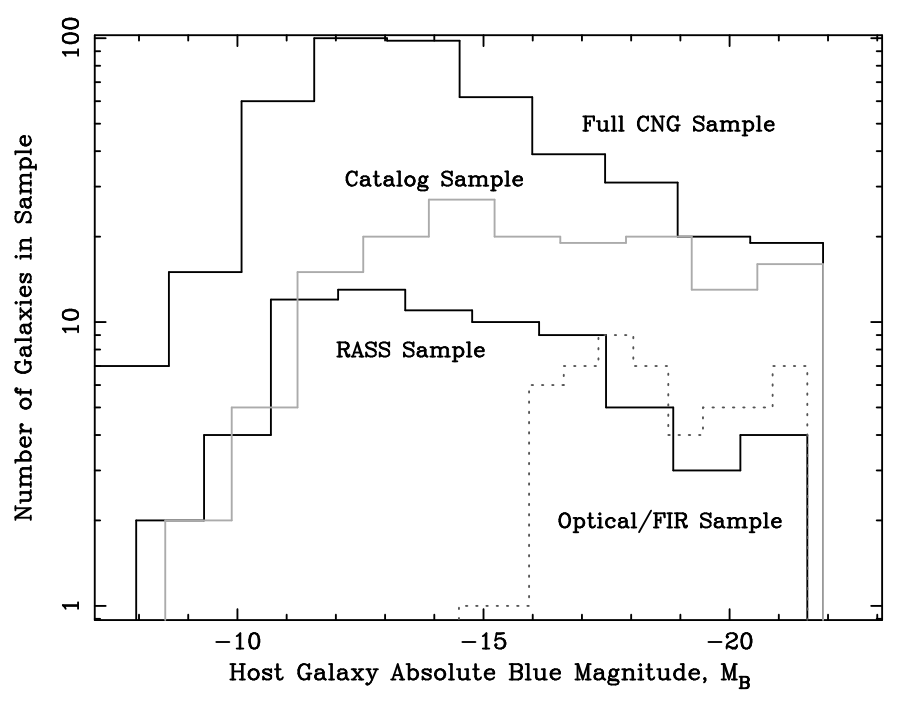

FIG. 1.- Distribution of the number of galaxies in the full Karachentsev et al. (2004) catalog (uppermost curve) as a function of $M_{B}$ and the distributions for three subsamples of the catalog as described in $\S \S 2.1$ (dotted historgram), 2.2 (lower heavy histogram), and 2.3 (light histogram).

In all samples, only galaxies in the CNG with tabulated masses are considered. This is a subset of 313 of the 451 galaxies in the CNG. For this subset, $\log \left(M / M_{\odot}\right)=$ $-0.39 M_{B}+2.65$. K04 define dwarf galaxies as those with $M_{B}>-17.0 \mathrm{mag}$ which corresponds to a mass $M<10^{9.3} \mathrm{M}_{\odot}$. Galaxy mass is computed by K04 from HI rotational velocity measurements (inclination-corrected HI line widths). Consequently, 93 of the 138 galaxies omitted in the subset are gas-poor early-type galaxies with revised Hubble type $T \leq 0$. This leaves 5 giant and $10 \mathrm{dwarf}$ early-tpe galaxies in the subset of 313 galaxies. The CNG sample of galaxies is shown as the uppermost histogram of Figure 2. The histogram for the subset of galaxies with tabulated masses is similar. As pointed out by K04, about $85 \%$ of galaxies in the local volume are dwarf galaxies although these galaxies contribute only $4 \%$ of the luminosity density. From the CNG subset, three subsamples are considered with different selection criteria and hence different potential biases:

\subsection{The optical and FIR flux-limited subsample}

This subsample contains all galaxies within the Uppsala Galaxy Catalog of (northern hemisphere) galaxies above the completeness limit of the UGC, $m_{p h}<14.5 \mathrm{mag}$ (Nilson 1973), with far-infrared (FIR) flux above the completeness limit of the Infrared Astronomy Satellite (IRAS) Point Source and Faint Source catalogs, $f_{\text {FIR }} \sim 1.5$ Jy (Beichman et al. 1988), and within $15 \mathrm{Mpc}$ of the Milky Way. There are 49 galaxies that meet these criteria among the 313 CNG subset. This subsample is biased toward the intrinsically optically-luminous galaxies with high gas (dust) content and hence relatively high current star-formation rates. Figure 1 shows that the subsample is, indeed, comprised of relatively luminous and hence massive galaxies but that there are a few nearby low-luminosity (low-mass) objects included as well.

\subsection{The nearby RASS subsample}

This subsample contains all galaxies within $4.0 \mathrm{Mpc}$ (a value based on expected count rates for ULXs, typical exposure times, and distances as tabulated by K04) located within $3^{\circ}$ of the centers of Rosat All Sky Survey (RASS) fields. These fields are $6.4^{\circ} \times 6.4^{\circ}$ regions observed in scanning mode by the ROSAT/PSPC with typical exposures of $\sim 400 \mathrm{~s}$ in typical galaxy-size regions of the sky. The RASS covers nearly the entire sky but the search algorithm used to query the data archivet uses circular search regions resulting in sampling only $70 \%$ of the field's total area (using a $3^{\circ}$ search radius). There are 107 galaxies in the CNG subset within $4.0 \mathrm{Mpc}$ and 72 or $67 \%$ in the subsample. This subsample contains the least bias of any of the subsamples. As shown in Figure 1, the distribution of blue luminosities for galaxies in this subsample most closely matches the CNG distribution.

\subsection{The X-ray source catalog subsample}

This subsample contains all galaxies in the CNG subset that have been imaged in the field of view of either ROSAT (PSPC or HRI), Chandra, or XMM-Newton pointed observations of sufficient exposure to detect ULXs and that have had their ULX populations identified through the literature. This includes many CNG subset galaxies that were targets of pointed observations and, in the case of ROSAT/PSPC with its $1^{\circ}$ radius FOV, several dwarf galaxies that were observed serendipitously. There are 155 galaxies in this subsample including 69 serendipitouslyobserved galaxies (those with large offsets from the observation's targeted aimpoint). This subsample should be considered an X-ray selected subsample since the majority of the galaxies were targets of pointed X-ray observations. It, like the Optical/FIR subsample (see Figure 1), is biased towards the larger galaxies which are the preferential targets of X-ray astronomy in general but it does contain a substantial number of dwarf galaxies.

\subsection{Sample X-ray Analysis}

Both the literature and available X-ray imaging data (limited to ROSAT, Chandra, and XMM-Newton) were searched for candidate ULXs in this study. In most cases, whenever a thorough analysis of existing data was previously reported through the refereed literature, we did not perform an independent study. In all cases, however, we did confirm that ULX candidates were within the angular area of the purported host galaxy and not coincident with its nucleus. For this, an initial search was made for candidate ULXs within a circle of radius $r=D_{25}$, i.e., extending to twice the optical semi-major axis of the galaxy. This allows for potential astrometric misalignments between reported X-ray source positions and galaxy locations as tabulated in the CNG and also for the large positional uncertainties that can occur in ROSAT and XMM-Newton data for weak sources because of their large point spread functions (PSFs). If candidate ULXs were identifed, then the spatial coincidence was reconfirmed using an elliptical approximation of the $D_{25}$ isophote [with parameters

\footnotetext{
${ }^{4}$ The High Energy Astrophysics Science Archive Research Center's on-line interface is http://heasarc.gsfc.nasa.gov/db-perl/W3Browse/w3browse.pl
} 
Optical/FIR Subsample

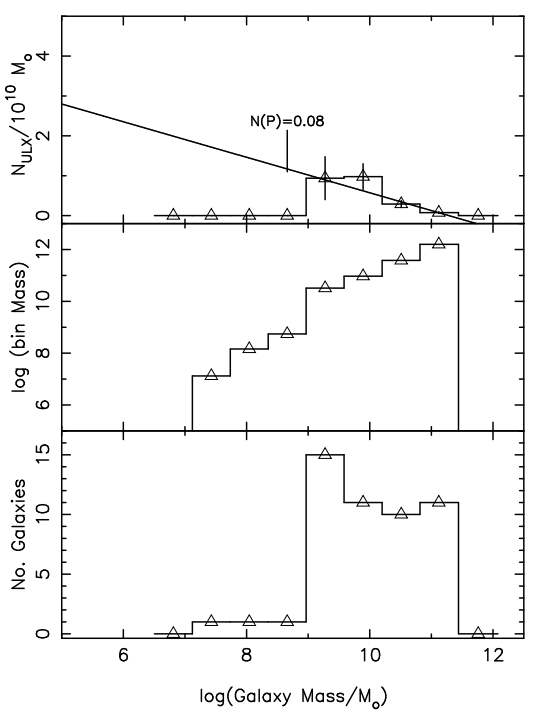

RASS Subsample

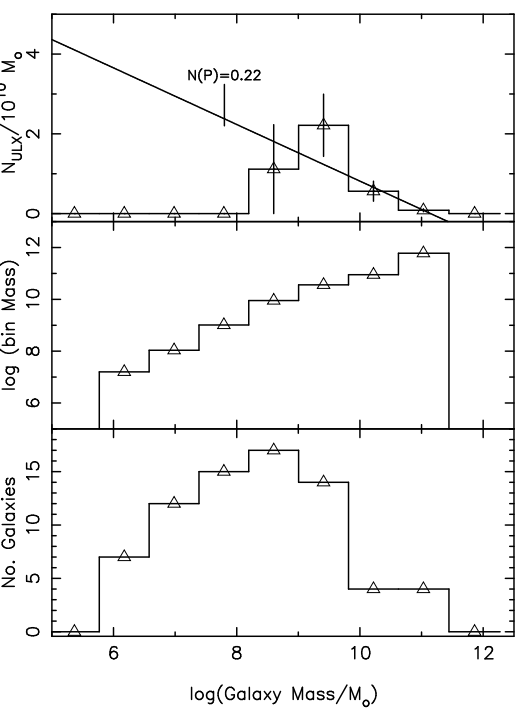

Catalog Subsample

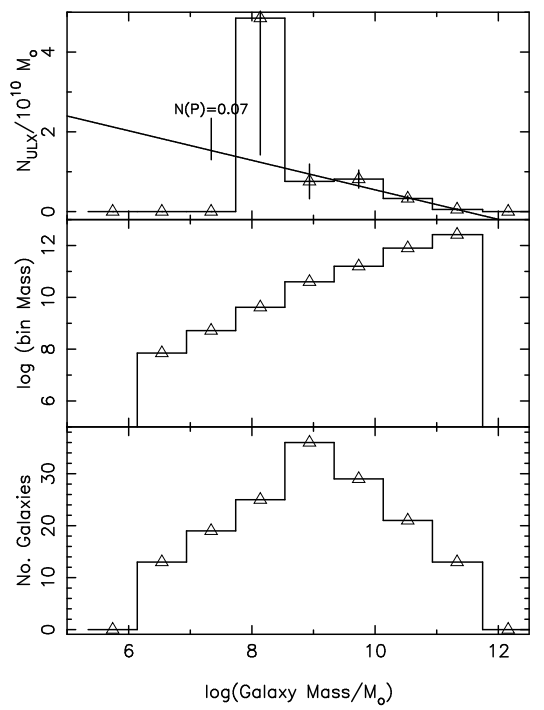

FIG. 2.- Histograms of the specific frequency of candidate ULXs (top panels), logarithm of the total mass in galaxies (middle panels), and number of galaxies (lower panels) against the logarithm of the host galaxy mass in solar mass units for three subsamples of galaxies tabulated in Karachentsev et al. (2004). From left to right, these subsamples are described in $\S \S 2.1,2.2$, and 2.3 . In all graphs, the highest and lowest mass bin are added for clarity of the plots; they contain zero galaxies. The lines shown in the upper panels are the best fit linear function to the data in bins with total mass exceeding $10^{10} \mathrm{M}_{\odot}$. In all cases, these are the highest 4 bins containing galaxies. Labels in the upper panels denote the number of ULX candidates predicted, $N(P)$, from extrapolation of the linear function to the next lower mass bin and scaling by the relative mass in that bin, $M_{10}$ (see text).

taken from the Third Reference Catalog of Bright Galaxies (de Vaucouleurs et al. 1991)], a check of the host galaxy position against the NASA/IPAC Extragalactic Database (NED) position (and references therein) and an evaluation of the X-ray source positional uncertainty. Sources coincident with host galaxy nuclei were rejected. This resulted in the exclusion of 10 nuclear sources; all are the wellknown active galactic nuclei of massive galaxies. Optical data (SDSS, DSS) were then visually inspected for potential foreground stars and background active galaxy counterparts which typically appear as bright and extended optical emitters, respectively. No such interlopers were identified using this test. A check was also made to ensure the entire $D_{25}$ isophote was imaged in the X-ray observation. The several large galaxies not fully covered by Chandra's FOV have been fully sampled by either ROSAT or XMM-Newton observations; thus no ULX candidates were overlooked.

The source-detection algorithm developed by Tennant (2006) was applied to X-ray images whenever an analysis of the X-ray point source population for a galaxy was not published or was of limited utility. All the checks for spatial coincidence with a host galaxy as outlined above were performed. X-ray fluxes were estimated from observed count rates within a $3 \sigma$ source radius (see Tennant 2006) using the Portable Interactive Multi-Mission Simulator (PIMMS; Mukai 1993) and assuming an absorbed power law source spectrum. The absorption was taken as the Galactic hydrogen column density along the line of sight to the host galaxy as tabulated from the HI map of Dickey \& Lockman (1990). The power-law index was assumed to be the average, $\Gamma=1.8$, obtained previously from single-component spectral model fits to a large number of ULX candidates by Swartz et al. (2004). X-ray luminosities were then estimated from the PIMMS fluxes and the distances tabulated by K04. The choice of a single, fixed shape, model spectrum is appropriate in the context of this study because only a small number of source photons $(\$ 100)$ were typically detected from candidate ULXs in those cases where thorough analysis was not previously reported.

Special mention must be made of the selection criterion of the RASS subsample $(\S 2.2)$ and the subsequent analysis results. As mentioned above, the distance limit criterion was chosen based on the expected count rate for ULXs (using PIMMS) and exposure times typical of the RASS and $\sim 10$ counts needed for detection (for each galaxy in this subsample, the actual exposure time at the location of the galaxy was determined from the exposure maps accompanying the RASS X-ray event lists). Our source-finding analysis of the RASS data did not detect 10 of 18 ULX candidates previously described in the literature (from pointed observations) and expected to be visible in the RASS according to this simple perscription. All non-detections are members of giant galaxies: Six are buried within the strong diffuse emission of the starburst galaxy M82, two are overlapping sources in RASS (though distinct in Chandra data) in NGC 4945, one is a heavilyabsorbed source in Circinus, and one is a weak source in NGC 7793 that must have faded compared to pointed ROSAT observations reported in Read \& Pietsch (1999). Thus, we attribute the non-detections to a combination of source confusion (aggravated by a rapidly-increasing offaxis PSF width; see Hasinger et al. 1994), high source column density (that decreases the detectable flux within the soft ROSAT passband from the PIMMS estimate assuming only a Galactic column density) and intrinsic source variability. For these sources, we included the flux values 
obtained from the literature.

\section{THE RESULTS}

A total of 5 dwarf galaxies $\left(M<10^{9.3} \mathrm{M}_{\odot}\right.$ corresponding to $M_{B}>-17 \mathrm{mag}$ ) were found to host a ULX in the present study. For comparison, there are a total of 35,19 , and 57 ULX candidates in the three (overlapping) subsamples described, respectively, in $\S \S 2.1-2.3$. Within these subsamples there are 10,58, and 91 dwarf galaxies, respectively. One new ULX candidate was discovered in a dwarf galaxy. This object was detected in the RASS image of E059-01 (PGC 21199), an IB(s)m dwarf of mass $1.7 \times 10^{9} \mathrm{M}_{\odot}$.

\subsection{The Specific ULX Frequency}

Histograms displaying the number of ULX candidates per unit mass, which we will refer to as the specific ULX frequency, against host galaxy mass were constructed for each subsample. The specific ULX frequency for the $i^{\text {th }}$ mass bin, $S_{i}^{u}$, is defined as $N_{i} / M_{10}^{i} \equiv \sum_{j}^{n_{i}} N_{j} / \sum_{j}^{n_{i}} M_{10}^{j}$ where $N_{j}$ is the number of ULX candidates detected in the $j^{\text {th }}$ galaxy, $M_{10}^{j}$ is that galaxy's mass in units of $10^{10} \mathrm{M}_{\odot}$, and the sum extends over all $n_{i}$ galaxies within the mass interval comprising the $i^{\text {th }}$ mass bin.

Figure 2 displays the $S_{i}^{u}$ histograms for all three subsamples (upper panels) the corresponding $\log \left(M_{10}^{i}\right)$ histograms (middle panels), and the number of galaxies, $N_{i}$, contained in each mass bin (lower panels). The $S_{i}^{u}$ statistical errors are $\left(N_{i}\right)^{1 / 2} M_{10}^{i}$. There are no ULX candidates detected in galaxies with mass less than $10^{8.5} \mathrm{M}_{\odot}$. There is also very little total mass, $M_{10}^{i}$, in low-mass bins in the subsamples (middle panels, Figure 2).

We can estimate the number of ULXs expected in these lower-mass bins: A simple linear function was fit to $S^{u}$ vs. $\log \left(M / M_{\odot}\right)$ over the mass bins with substantial total mass, $M_{10}^{i} \geq 1$, in each subsample. There are 4 such bins, spanning roughly 3 decades in host galaxy mass, in each subsample. From this, the number of ULXs predicted, $N_{i}(P)$ (where $P$ denotes predicted), in the $i^{t h}$ lower-mass bin is estimated by extrapolating this function and weighting by the relative mass: $N_{i}(P)=f_{i}\left(S^{u}\right) M_{10}^{i}$ where $f_{i}\left(S^{u}\right)$ is the fitting function evaluated at the $i^{t h}$ mass bin. Values for $N_{i}(P)$ are given in the upper panels of Figure 2 along with the best-fitting function, $f_{i}\left(S^{u}\right) . N_{i}(P) \ll 1$ in all cases because $M_{10}^{i} \ll 1$ for these lower-mass bins. That is, there is simply too little mass in low-mass dwarf galaxies to expect to find ULXs.

The fitting function used here was chosen to maximize the number of ULXs predicted in lower-mass bins and hence provide a conservative expectation value. There is no a priori reason to expect the specific frequency of ULXs to increase with decreasing host galaxy mass (a more intuitive expectation is that $S^{u}$ is independent of mass but it is clear from Figure 2 that $S^{u}$ equals a constant is a poor representation of the data).

\subsection{The ULX Frequency \& The Star Formation Rate}

We do, however, expect the number of ULX candidates to increase for galaxies with high current star formation rates (Grimm et al. 2003; Swartz et al. 2004; Colbert et al. 2004). In fact, from a sample of starburst galaxies, Grimm et al. (2003) find the number of X-ray sources above a luminosity, $L$, depends linearly on the current star formation rate, $R_{S F R}$, as: $N(>L)=1.32 R_{S F R}\left(L_{39}^{-0.61}-21.0^{-0.61}\right)$ where $R_{S F R}$ is measured in units of $\mathrm{M}_{\odot} \mathrm{yr}^{-1}$ and $L_{39}$ is the X-ray luminosity in units of $10^{39} \mathrm{ergs} \mathrm{s}^{-1}$. Thus, we expect 1.1 ULXs per unit SFR.

The SFR for galaxies with a sizeable dust opacity can be estimated from its FIR luminosity: $R_{S F R}=$ $4.5 \times 10^{-44} L_{F I R} \mathrm{M}_{\odot} \mathrm{yr}^{-1}$ (e.g., Kennicutt 1998). Here, $L_{F I R}$ is the FIR luminosity estimated from the IRAS $60 \mu \mathrm{m}$ and $100 \mu \mathrm{m}$ flux densities, $L_{F I R} / 4 \pi D^{2}=1.26 \times$ $10^{-11}\left(2.58 S_{60}+S_{100}\right) \operatorname{ergs~s}^{-1}$. We have computed the star formation rate for the galaxies in the optical and FIR fluxlimited subsample ( $\S 2.1$; many of the CNG galaxies are not included in the IRAS catalogs so we cannot make this computation for the other subsamples) and constructed histograms of the distribution of ULXs with host galaxy mass as before but with $M_{10}^{j}$ replaced with $R_{S F R}^{j}$ in the sums, $\sum_{j}^{n_{i}} N_{j} / \sum_{j}^{n_{i}} R_{S F R}^{j}$, over the $n_{i}$ galaxies contained in the $i^{t h}$ mass bin.

Figure 3 shows this distribution. The ULX frequency normalized to unit SFR is roughly a constant for the bins with substantial total mass. The best fit constant value is $2.3 \pm 0.7$ which is about $2 \sigma$ above the value predicted by Grimm et al. (2003). A fit to a linear function is not a significant improvement according to an F-test. Hence, the number of ULXs per unit SFR is similar for dwarf and giant galaxies in this subsample. However, the SFR per unit mass is higher by about a factor of 3 for the dwarf galaxies compared to the giants which is consistent with the trend in specific ULX frequency deduced above.

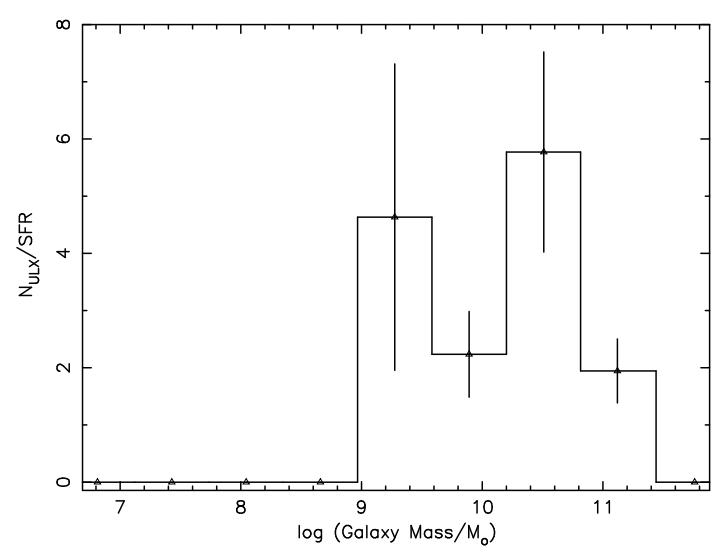

FIG. 3.- Histogram of the number of candidate ULXs per unit star formation rate $\left(\mathrm{M}_{\odot} \mathrm{yr}^{-1}\right)$ against the logarithm of the host galaxy mass (in solar mass units) for the galaxy subsample described in $\S 2.1$. Total galaxy mass in each bin and number of galaxies per bin are the same as in the lower two left-most panels of Figure 2.

\subsection{Bias Assessment}

There are three catagories of X-ray sources that may occur more or less frequently in dwarf compared to giant galaxies and thus have the potential to introduce biases in the present study: Contaminating background sources, transient ULXs, and nuclear sources. Assuming the spatial distribution of background sources is uniform across the sky, the relative contributions of background sources to the number of ULX candidates in each mass bin is proportional to the sum of the areas within the optical extents 
of the galaxies included in the bin. This is strictly true only for galaxies at a fixed distance because the number of background sources per unit area of sky increases with decreasing source flux and the search for ULXs extends to lower fluxes for more distant galaxies. However, the mean distance to dwarf galaxies in any of the subsamples (and to the CNG subset with masses defined) is less than that to giant galaxies which implies that potential contamination by background sources per unit area is higher for the higher-mass bins considered. Furthermore, the total sky area covered by galaxies in each mass bin increases towards higher-mass bins so, again, the potential contamination is higher for the higher-mass bins. Thus, any bias introduced by background sources applies in the opposite sense to the trend determined here, namely, that the specific frequency of ULXs increases with decreasing galaxy mass.

The probability of detecting transient sources increases with the rate of observations sampling a particular target galaxy. The present study does not differentiate between transient and non-transient ULX candidates but it can be surmised that the giant galaxies in the Local Volume are more apt to have been observed multiple times over the course of the decades spanned by the ROSAT, Chandra, and XMM-Newton missions than are the dwarf galaxies. Thus, any bias introduced by ULX transience would favor an increase in the number of ULX candidates discovered in giant over dwarf galaxies.

The nuclear regions of sample galaxies where not a priori excluded from our search for ULX candidates. However, $\mathrm{X}$-ray luminous active galactic nuclei may hide nearby ULXs in their bright glow, effectively imposing an exclusion region in the area of highest galactic surface mass density. As the brightest AGN are associated with the most massive galaxies, ULXs hidden by AGN brilliance could lead to an underestimate of the ULX population in the higher-mass bins. This is further exacerbated by the fact that the radial distribution of ULXs in general peaks toward the centers of galaxies (Swartz et al. 2004; Liu et al. 2006). A total of 18 galaxies in the present study are listed as quasars or AGN in the catalog of Veron-Cetty \& Veron (2006). Of these, 11 host X-ray bright nuclear sources (all are giant galaxies). Fortunately, all have been observed with Chandra so that a careful evaluation of the nuclear regions of these galaxies at high angular resolution could be made. No evidence was found for hidden ULXs; thus, no bias is apparent in the present results due to nuclear sources.

\section{DISCUSSION}

Ultraluminous X-ray sources occur in dwarf galaxies in the Local Volume; at least down to galaxy masses of $\sim 3 \times 10^{8} \mathrm{M}_{\odot}$. In fact, for the galaxies studied here, the specific frequency of ULXs in low-mass galaxies is higher than in massive giants. This trend in the population of ULXs is unanticipated.

Still, ULXs in dwarf galaxies are rare, of order 1 ULX occurs per $10^{10} \mathrm{M}_{\odot}$ in the galaxies studied here, which means only one in $10010^{8} \mathrm{M}_{\odot}$ dwarf galaxy is expected to host a ULX. Five ULX candidates were identified in 118 dwarf galaxies $\left(M<10^{9.3} \mathrm{M}_{\odot}\right.$ corresponding to $\left.M_{B}>-17 \mathrm{mag}\right)$ in the present study. Assuming the sample of galaxies was randomly selected, the Gehrels statistic (Gehrels 1986) gives a 90\% confidence that the true mean exceeds 2.4. Thus it is unlikely that our result is purely a statistical fluctuation. We detected no ULXs in galaxies with mass $<10^{8.5} \mathrm{M}_{\odot}$ but, if the trend continues to lower masses, then we expect $\leq 0.22$ ULXs to have been detected in galaxies in our sample with mass $\sim 10^{7.8} \mathrm{M}_{\odot}$ which is consistent with finding 0 .

It is worthwhile to consider what physical differences exist between dwarf and giant galaxies that could give rise to the observed trend. Past studies have concluded that a strong correlation exists between star formation rate (SFR) and the number and luminosity density of ULXs (Swartz et al. 2004; Liu et al. 2006; though see Ptak \& Colbert 2004). For the one subsample purposefully chosen here to include FIR-luminous galaxies, the specific current SFR in dwarfs is larger by a factor of 3 than in giants. Thus, an increase in the specific ULX frequency in dwarf galaxies relative to giants would be expected at least for this subsample.

There are also, potentially, differences in the stellar evolution and star formation processes in nearby dwarf galaxies compared to the giants that may favor the formation of ULXs. Dwarf galaxies tend to evolve more slowly than giants hence their metallicity at the current epoch is systematically lower than that of giants (Lee et al. 2006). Massive stars of low metallicity loose less mass through winds than do high- $Z$ stars and leave higher-mass compact objects following core collapse (Heger et al. 2003). Higher mass black hole remnants, in turn, can radiate at higher luminosities without violating the Eddington limit. Low- $Z$ donor stars may transfer more mass in Roche lobe overflow, and/or over a longer period of time, than do high- $Z$ donors with high wind mass loss rates. This would allow higher luminosity and longer-lived ULXs to arise in low-Z systems.

Dwarf galaxies form massive star clusters at a rate higher than expected for their size. Billet et al. (2002) explain this as due to a lack of shear in dwarf galaxies that tends to fragment large molecular clouds and prevent formation of large stellar clusters in spiral galaxies. There may be a connection between an excess of massive compact clusters and an excess of ULXs in dwarf galaxies. Both objects may be the end products of rapid collapse of molecular clouds with the ULXs formed from the most massive stars created in the collapse. Gravitational coalescence of intermediate-mass protostars to form massive stars is observed in Galactic protoclusters (Peretto et al. 2007). The cold initial conditions (low turbulent energy relative to gravitational energy) needed for protostar merger are the same conditions envisioned by Billet et al. (2002) to explain the formation of massive clusters in dwarf galaxies. These conditions may result from rapid compression by external processes caused by galactic-scale interactions (e.g., Keto et al. 2005) - processes that are more effective in dwarfs because of their smaller size. Interestingly, Billet et al. (2002) conclude that the smallest galaxies cannot sample the high end of the cloud mass spectrum and that they will fall short of producing massive compact clusters. Perhaps a similar cutoff occurs in the production of ULXs and they are lacking in very low-mass galaxies.

We have determined that ULXs occur in dwarf galaxies and that their specific frequency increases with decreasing host galaxy mass. This suggests that there may be special (though not unique) environmental conditions in 
dwarf galaxies that preferentially lead to the formation of ULXs. This result should be addressed by theoretical models proposed to explain the ULX phenomenon.

This work was supported, in part, by Chandra Award
GO6-7081A issued by the Chandra X-ray Observatory Center which is operated by the Smithsonian Astrophysical Observatory for and on behalf of NASA under contract NAS8-03060.

\section{REFERENCES}

Beichman, C. A., Neugebauer, G., Habing, H. J., Clegg, P. E., Chester, T. J. 1988, NASA RP-1190 (Washington, DC:GPO)

Billet, O. H., Hunter, D. A., \& Elmegreen, B. G. 2002, AJ, 123, 1454

Colbert, E. J. M., Heckman, T. M., Ptak, A. F., Strickland, D. K., \& Weaver, K. A. 2004, ApJ, 602, 231

de Vaucouleurs G., de Vaucouleurs A., Corwin Jr. H. G., Buta R. J., Paturel G., \& Fouque P. 1991, Third Reference Catalogue of Bright Galaxies (NewYork: Springer-Verlag)

Dickey, J. M., \& Lockman, F. J. 1990, ARA\&A, 28, 215

Gao, Y., Wang, Q. D., Appleton, P. N., \& Lucas, R. A. 2003, ApJ, 596, L171

Geha, M., Blanton, M. R., Masjedi, M., \& West, A. A. 2006, ApJ, 653,240

Gehrels, N. 1986, ApJ, 303, 336

Gilfanov, M., Grimm, H. -J., \& Sunyaev, R. 2004, MNRAS, 347, L57

Grimm, H. -J., Gilfanov, M., \& Sunyaev, R. 2003, MNRAS, 339, 793

Haines, C. P., Gargiulo, A., La Barbera, F., Mercurio, A., Merluzzi, P., \& Busarello, G. 2007, MNRAS, in press (arXiv:0707.1262 1 )

Hasinger, G. et al. 1994, Legacy 4, 40 (CAL/ROS/93-015)

Heger, A., Fryer, C. L., Woosley, S. E., Langer, N., \& Hartmann, D. H. 2003, ApJ, 591, 288

Hunter, D. A., \& Gallagher, J. S. 1986, PASP, 98, 5
Irwin, J. A., Bregman, J. N., \& Athey, A. E. 2004, ApJ, 601, L143

Karachentsev, I. D., Karachentseva, V. E., Huchtneier, W. K., \& Makarov, D. L. 2004, AJ, 127, 2031

Kennicutt, R. C., Jr. 1998, ARA\&A, 36, 189

Keto, E., Ho, L. C., \& Lo, K. -Y. 2005, ApJ, 635, 1062

King, A. R. 2004, MNRAS, 347, L18

Lee, H., et al. 2006, ApJ, 647, 970

Lehmer, B. D., et al. 2006, AJ, 131, 2394

Liu, J. -F., Bregman, J. N., \& Irwin, J. 2006, ApJ, 642, 171

Mukai, K. 1993, Legacy, 3, 21

Nilson, P. 1973, Uppsala General Catalogue of Galaxies (Uppsala: Astronomiska Observatorium)

Peretto, N., Hennebelle, P., \& André, P. 2007, A\&A, 464, 983

Ptak, A. \& Colbert, E. 2004, ApJ, 606, 291

Read, A. M. \& Pietsch, W. 1999, A\&A, 341, 8

Swartz, D. A., Ghosh, K. K., Tennant, A. F., \& Wu, K. 2004, ApJS, 154,519

Tennant, A. F. 2006, AJ, 132, 1372

Winter, L. M., Mushotzky, R. F., \& Reynolds, C. S. 2006, ApJ, 649, 730

Zezas, A., \& Fabbiano, G. 2002, ApJ, 577, 726 